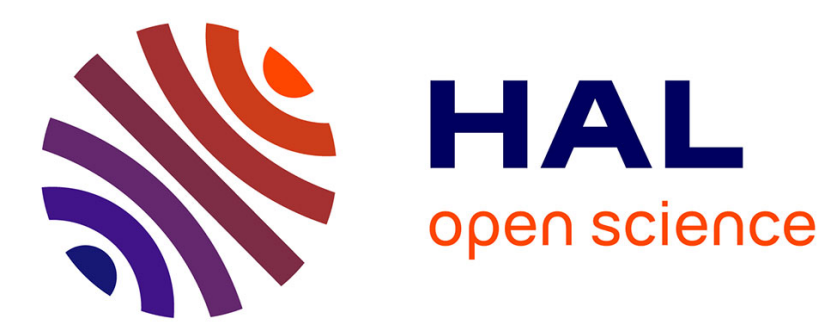

\title{
Sylvopastoralisme dans le Haut-Verdon
}

Gérard Decaix

\section{To cite this version:}

Gérard Decaix. Sylvopastoralisme dans le Haut-Verdon. Revue forestière française, 1994, 46 (S), pp.49-58. 10.4267/2042/26617 . hal-03444509

\section{HAL Id: hal-03444509 \\ https://hal.science/hal-03444509}

Submitted on 23 Nov 2021

HAL is a multi-disciplinary open access archive for the deposit and dissemination of scientific research documents, whether they are published or not. The documents may come from teaching and research institutions in France or abroad, or from public or private research centers.
L'archive ouverte pluridisciplinaire HAL, est destinée au dépôt et à la diffusion de documents scientifiques de niveau recherche, publiés ou non, émanant des établissements d'enseignement et de recherche français ou étrangers, des laboratoires publics ou privés. 


\section{SYLVOPASTORALISME DANS LE HAUT-VERDON}

\section{G. DECAIX}

Du Verdon, tout le monde connaît les vertigineuses gorges qui entaillent les calcaires de HauteProvence. Mais, si on se donne la peine de remonter le cours de cet impétueux affluent de la Durance, on découvrira rapidement un tout autre paysage : la vallée s'élargit en vastes cuvettes, les sommets s'élèvent jusqu'à culminer au-dessus de $2000 \mathrm{~m}$, le climat et la végétation prennent une tonalité montagnarde.

Cet ensemble qui forme le lien entre le pays méditerranéen et la haute montagne alpine, autrefois très peuplé et parcouru d'immenses troupeaux, ne s'anime plus qu'en période estivale. Depuis une quinzaine d'années, dans un souci de gestion de l'espace rural, l'Office national des Forêts, en collaboration avec de nombreux partenaires, y mène des opérations de rénovation d'anciens parcours ovins, progressivement colonisés par la lande et le Pin sylvestre.

Après une description rapide du contexte régional, nous examinerons plus en détail l'exemple de remise en valeur sylvopastorale de La Colle Saint-Michel sur la commune de ThorameHaute, qui est entrée dans sa phase de gestion depuis 1988. Nous le complèterons par des observations faites sur d'autres opérations menées depuis dans les communes voisines, pour essayer, en conclusion, de dégager les éléments de réussite de tels projets.

\section{LE CONTEXTE PHYSIQUE ET HUMAIN. LES PROBLĖMES DE GESTION}

\section{Le milieu naturel}

Le climat, de type montagnard marqué d'influence méditerranéenne, peut paraître modéré au regard des moyennes (précipitations de 1000 à $1200 \mathrm{~mm}$, températures moyennes de $8^{\circ} \mathrm{C}$ ). En fait, celles-ci cachent une grande irrégularité inter et intra-annuelle défavorable à la végétation, avec un important déficit hydrique estival.

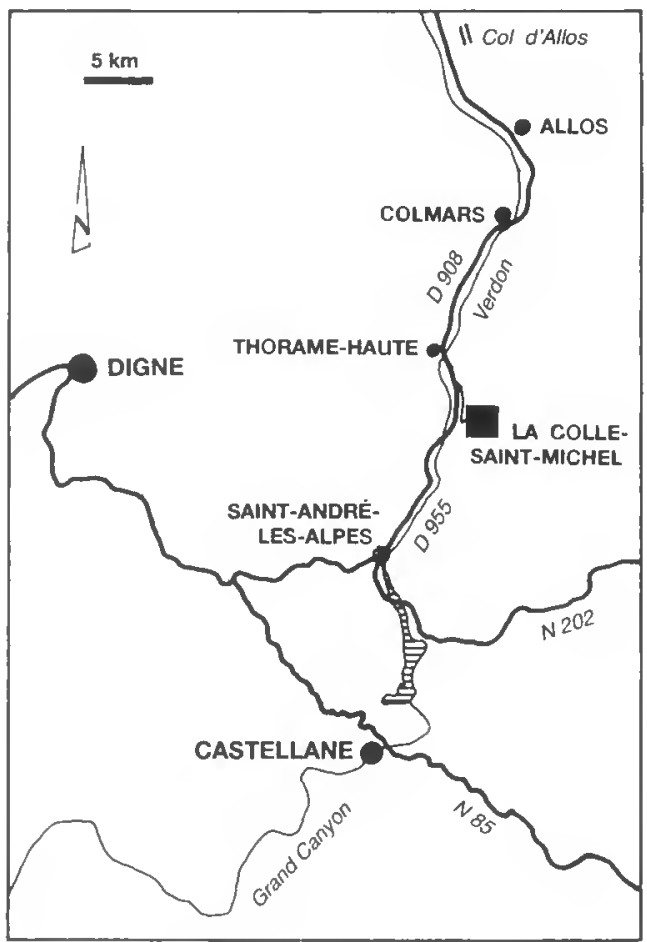


Le substrat est constitué pour l'essentiel par des marno-calcaires alternant en bancs d'épaisseur variable et donnant naissance à des éboulis cryoclastiques ou à des éluvions peu épais recouvrant les formations en place.

La végétation se différencie avec l'altitude et l'exposition. À l'étage montagnard, les versants secs sont occupés par la lande à Genêt cendré ou à Buis, plus ou moins arborée, alors que les ubacs et les zones de faible pente sont le domaine de la forêt de Pin sylvestre surtout et de Sapin ou de Hêtre. Au subalpin, si la pelouse domine encore, elle est progressivement envahie par le Pin sylvestre et le Mélèze.

Dans cet ensemble, ce sont les zones de topographie les plus douces (piémont et croupes sommitales arrondies), entre 1000 et $1600 \mathrm{~m}$ d'altitude, anciennement parcours ovins, aujourd'hui colonisées par la lande et la pinède sylvestre, qui nous intéressent dans le cadre de la remise en valeur sylvopastorale.

\section{Les activités humaines}

Jusqu'à l'aube du $\mathbf{X X} \mathbf{X}^{\mathbf{e}}$ siècle, la population est importante. L'agriculture, et en particulier l'élevage, sont la base des activités d'un monde presque strictement rural, au point de structurer complètement l'espace et de reléguer la forêt à quelques ubacs inaccessibles.

Avec le $\mathbf{X X}$ siècle, l'exode rural commence et s'amplifie après la Première Guerre mondiale. La mise en valeur du territoire évolue profondèment. D'abord à travers les reboisements induits par la politique de restauration des terrains en montagne (RTM), puis avec la régression du pastoralisme, par l'embroussaillement et la reforestation naturelle de vastes espaces abandonnés par les troupeaux.

Aujourd'hui, l'élevage, même profondément modifié, reste un élément important de l'économie locale. Mais la forêt a acquis une place non négligeable, malgré des potentialités de production moyenne et une filière bois réduite et fragile. Récemment, la fonction sociale de tous ces espaces a pris une importance prépondèrante à travers l'accueil d'un tourisme aux activités de plus en plus diversifièes et de leur rôle paysager. Enfin, la préservation de richesses écologiques, souvent ignorées au profit d'espaces plus prestigieux, devient une préoccupation grandissante.

\section{Les problèmes de gestion}

Dans ce contexte, la gestion des anciens parcours aujourd'hui colonisés par la lande et le Pin sylvestre se heurte à de nombreux problèmes de natures variées :

La fermeture de l'espace entraîne:

- une profonde modification du paysage qui, perdant ses lignes de force, se banalise ;

- une sous-exploitation pastorale généralisée mais, paradoxalement, une surexploitation localisée des dernières pelouses pouvant aller jusqu'à leur destruction ;

- un risque accru vis-à-vis de l'incendie ;

- un appauvrissement de la biodiversité.

L'hétérogénéité des boisements, leur productivité réduite et la qualité moyenne des bois jamais éclaircis n'autorisent que rarement une sylviculture classique basée sur des prélèvements réguliers mais trop faibles.

La structure foncière est un blocage à de nombreux projets et, dans ces conditions, l'importance de la propriété communale est souvent un atout prépondèrant.

Le développement touristique et le maintien d'une certaine biodiversité sont à intégrer dans tout projet de mise en valeur. 
Colonisation des anciens

parcours ovins

par le Pin sylvestre.

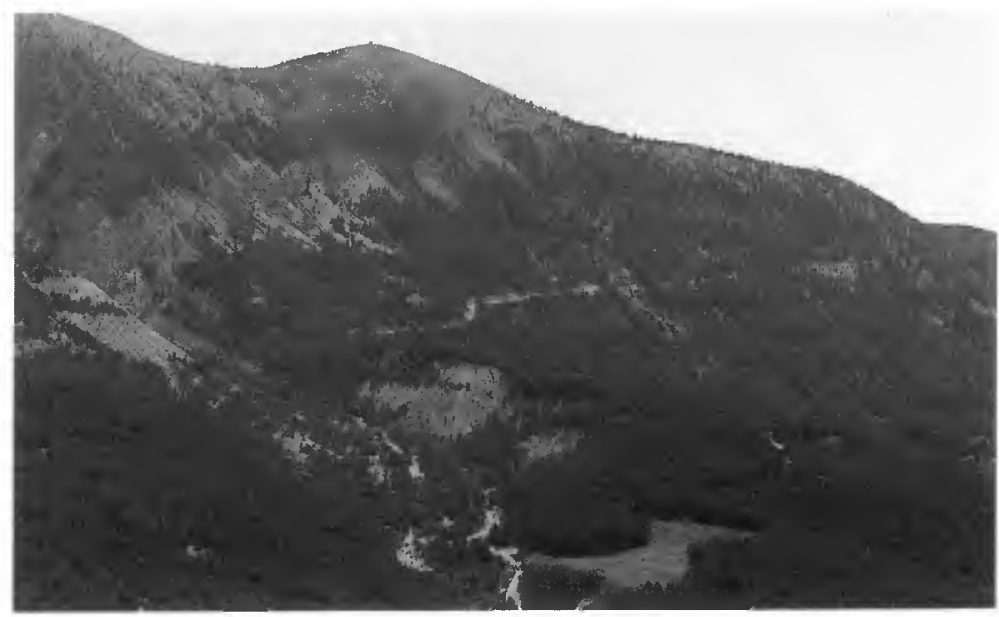

Boisement naturel de Pin sylvestre.

Photos G. DECAIX

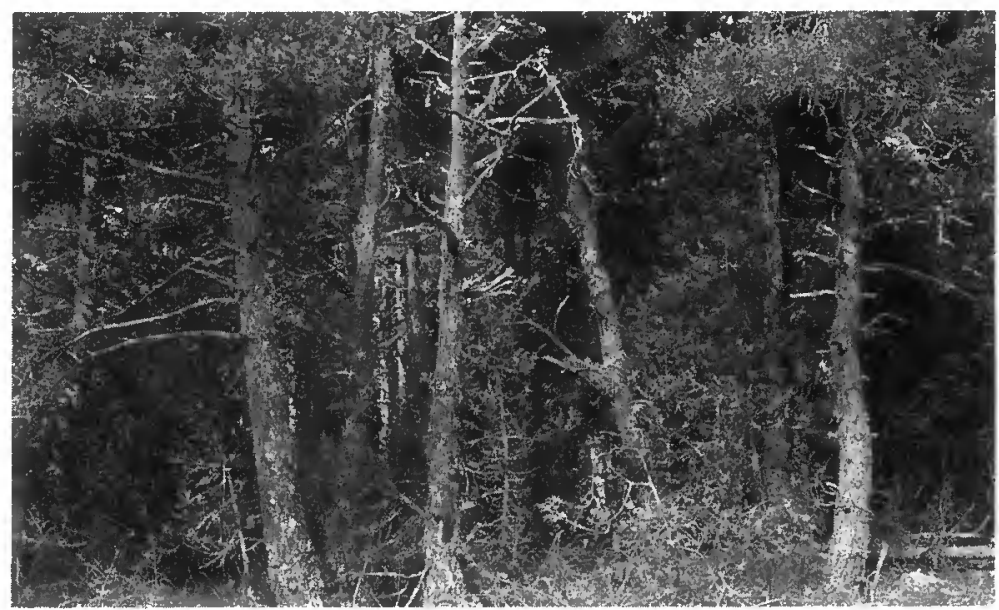

Dans ces conditions, l'aménagement sylvopastoral peut apparaître comme une solution intéressante à plusieurs titres : mise en valeur forestière et pastorale bien entendu, mais aussi amèlioration du paysage, maintien de la biodiversité et intégration des aménagements touristiques.

\section{L'EXEMPLE DE L'AMÉNAGEMENT SYLVOPASTORAL DE LA COLLE SAINT-MICHEL (commune de Thorame-Haute)}

En 1982, la commune de Thorame-Haute, soucieuse de mettre en valeur ses anciens parcours, soumet au régime forestier près de 700 ha, "sous réserve" d'y mettre en place un aménagement intégrant les objectifs pastoraux, forestiers et touristiques (ski de fond et randonnées pédestres). L'ONF, nouveau gestionnaire, s'entoure aussitôt d'organismes complétant ses propres compètences : I'INRA-Écodéveloppement, le CEMAGREF de Montpellier, le CERPAM $04{ }^{~(1)}$.

(1) CERPAM : Centre d'Études et de Recherche pastorale Alpes-Méditerranée. 


\section{G. DECAIX}

\section{La phase expérimentale}

En 1983, le CEMAGREF va tester, sur des placettes de référence, une première conception du traitement consistant à :

- débroussailler les zones de landes;

- éclaircir de façon uniforme les peuplements de Pin sylvestre (de 30-60 ans, atteignant $15 \mathrm{~m}$ de haut et 2000 à 3000 tiges/ha) à la densité moyenne de $3 \mathrm{~m} \times 3 \mathrm{~m}$ et $4 \mathrm{~m} \times 4 \mathrm{~m}$, élaguer les tiges restantes à $2 \mathrm{~m}$ de haut, évacuer les rondins de la coupe et broyer broussailles et rémanents préalablement mis en andains.

Le tableau I (p. 53) résume le coût de chacune des interventions.

Cette première phase a permis de tirer certaines conclusions :

- Les coûts très élevés (surtout enstérage et broyage) ne permettent pas d'envisager le traitement de grandes surfaces.

- La sélection des tiges ne permet pas une véritable amélioration du peuplement, tandis que les effets de l'éclairement au sol sont insuffisants pour que s'installent de véritables espèces fourragères.

- La commercialisation des bois sur pied est aléatoire à cause du volume hectare faible et de difficultés de débardage.

Aussi, la conception du traitement a fortement évolué : à la superposition forêt-pelouse a été substituée la juxtaposition de parquets de 5 à $10 \mathrm{ha}$, les uns plus strictement forestiers, les autres pastoraux. Cette conception a permis d'intégrer plus facilement les besoins touristiques en créant des "couloirs" où se sont insérées les pistes de ski de fond.

\section{La phase de rénovation}

L'extension de ce traitement sur une centaine d'hectares a nécessité une description initiale des milieux, puis la définition précise des différentes interventions. Devant l'extrême diversité d'un espace en pleine évolution, seulement trois types de milieux ont été retenus et cinq traitements définis visant à l'amélioration et à l'augmentation de la ressource pastorale (traitements $A, B$ et C) ou forestière ( $D$ et $E$ ). Préalablement, un réseau de $15 \mathrm{~km}$ de pistes d'exploitation, adaptées à la pratique du ski de fond, a été élaboré.

Le tableau II (p. 53) résume l'ensemble de ces mesures, tandis que les cartes 1 et 2 (p. 54) en donnent l'extension spatiale. On peut en tirer les conclusions suivantes :

- le coût total à l'hectare traité atteint $5800 \mathrm{~F}$ (et s'abaisse à $4700 \mathrm{~F}$ si on exclut la desserte) nettement inférieur aux $21600 \mathrm{~F}$ de la phase expérimentale ;

- la recette atteint $400 \mathrm{~F} / \mathrm{ha}$ et le bilan se situe donc à un coût de $5200 \mathrm{~F} / \mathrm{ha}$ au lieu de $17100 \mathrm{~F}$;

- le coût de débroussaillement est raisonnable (1 $800 \mathrm{~F} / \mathrm{ha}$ ), alors que l'incinération des rémanents et la remise en état de l'ancienne coupe alourdissent les dépenses.

\section{La phase de gestion}

Le modèle de gestion doit permettre de concilier les activités pastorales, forestières et touristiques en améliorant le potentiel de production. Il comprend deux volets, l'un forestier, l'autre pastoral, avec pour chacun des mesures imposées par l'exploitation touristique du site.

L'aménagement forestier crée une véritable série de production sylvopastorale visant à l'amélioration quantitative et qualitative des productions pastorales et forestières. II préconise un traitement 
Tableau I

\begin{tabular}{|c|c|c|}
\hline Opération & Pourcentage du temps & Coût à l'hectare \\
\hline $\begin{array}{l}\text { Abattage-façonnage } \ldots \ldots \ldots \ldots \ldots \ldots \ldots \\
\text { Ėlagage } \ldots \ldots \ldots \ldots \ldots \ldots \ldots \ldots \ldots \\
\text { Enstérage des billons, mise en andains de produits } \\
\text { d'élagage, débroussaillage et rémanents } \ldots \ldots \ldots \ldots\end{array}$ & $\begin{array}{l}24 \\
12\end{array}$ & $\begin{array}{l}3400 \mathrm{~F} \\
1700 \mathrm{~F} \\
8500 \mathrm{~F}\end{array}$ \\
\hline Broyage mécanique des andains $\ldots \ldots \ldots \ldots \ldots$ & 4 & $8000 \mathrm{~F}$ \\
\hline \multicolumn{2}{|c|}{ 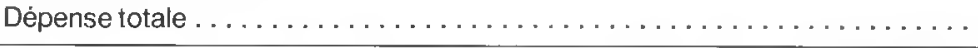 } & $21600 \mathrm{~F}$ \\
\hline \multicolumn{2}{|c|}{ 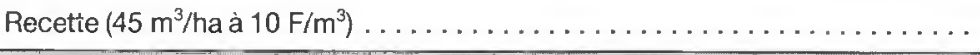 } & $-4500 \mathrm{~F}$ \\
\hline \multicolumn{2}{|c|}{ Coût total $\ldots \ldots \ldots+\ldots \ldots \ldots \ldots \ldots \ldots \ldots \ldots \ldots$} & $17100 \mathrm{~F}$ \\
\hline
\end{tabular}

Tableau II

\begin{tabular}{|c|c|c|c|c|c|c|c|}
\hline $\begin{array}{l}\text { Type de milieu } \\
\text { (surface/ha) }\end{array}$ & Traitement & $\begin{array}{l}\text { Surface } \\
\text { traitẻe } \\
\text { (ha) }\end{array}$ & Travaux et coupes & Coût total & $\begin{array}{l}\text { Coût/ha } \\
\text { traité }\end{array}$ & $\begin{array}{l}\text { Revenu } \\
\text { total }\end{array}$ & $\begin{array}{l}\text { Revenu } \\
\text { /ha traité }\end{array}$ \\
\hline $\begin{array}{l}\text { Pelouse plus ou moins } \\
\text { embroussaillée ( } 17 \mathrm{ha})\end{array}$ & A & 17 & $\begin{array}{l}\text { Débroussaillement méca- } \\
\text { nique gyrobroyeur à axe } \\
\text { vertical à chaînes. } \\
\text { Élagage de } 1,5 \mathrm{~m} \text { et mise } \\
\text { en tas des branches. } \\
\text { Brûlage des tas. }\end{array}$ & $\begin{array}{l}30600 \\
43800 \\
10000\end{array}$ & $\begin{array}{r}1800 \\
2576 \\
810\end{array}$ & & \\
\hline \multirow[t]{3}{*}{$\begin{array}{l}\text { Boisement de Pins syl- } \\
\text { vestres ( } 59 \text { ha). } \\
40 \text { à } 60 \text { ans. } \\
2000 \text { à } 3000 \text { tiges/ha. }\end{array}$} & B & 9 & $\begin{array}{l}\text { Coupe rase } \\
\text { - Mise en tas et brûlage } \\
\text { des rémanents. } \\
\text { Débroussaillement. }\end{array}$ & $\begin{array}{l}18000 \\
16200\end{array}$ & $\begin{array}{l}2000 \\
1800\end{array}$ & & \\
\hline & D & 32 & $\begin{array}{l}\text { Coupe d'amélioration } \\
\text { - vendue sur } 17 \text { ha. } \\
\text { - délivrée gracieuse- } \\
\text { ment sur } 10 \text { ha. } \\
\text { - exploitée onéreuse- } \\
\text { ment sur } 5 \text { ha. }\end{array}$ & 29500 & $\begin{array}{r}0 \\
0 \\
5900\end{array}$ & 42250 & 2485 \\
\hline & Non traité & 19 & & & & & \\
\hline \multirow[t]{5}{*}{$\begin{array}{l}\text { Ancienne coupe ( } 28 \text { ha) } \\
\text { datant de } 15 \text { ans. } \\
60 \text { à } 100 \text { ans. } \\
100 \text { à } 150 \text { tiges/ha. }\end{array}$} & C & 14 & $\begin{array}{l}\text { Nettoyage (élagage à } \\
1,5 \mathrm{~m} \text {, abattage, démon- } \\
\text { tage des houppiers, mise } \\
\text { en tas, incinération, dè- } \\
\text { broussaillage-gyrobroyeur } \\
\text { et débroussailleuse } \\
\text { portée). }\end{array}$ & 70000 & 5000 & & \\
\hline & $E$ & 14 & Plantation (1) & 184000 & 13180 & & \\
\hline & & 86 & $\begin{array}{l}\text { Total travaux syluopas- } \\
\text { toraux }\end{array}$ & 402100 & 4674 & & \\
\hline & & & $\begin{array}{l}\text { Desserte piste en } 3 \mathrm{ml} \text { de } \\
\text { large }\end{array}$ & 100000 & & & \\
\hline & & & Total & 502100 & & & \\
\hline
\end{tabular}

(1) En potets de Mèlèze et Sapin en godet à la densité de 1100 plants/ha. 
G. DECAIX

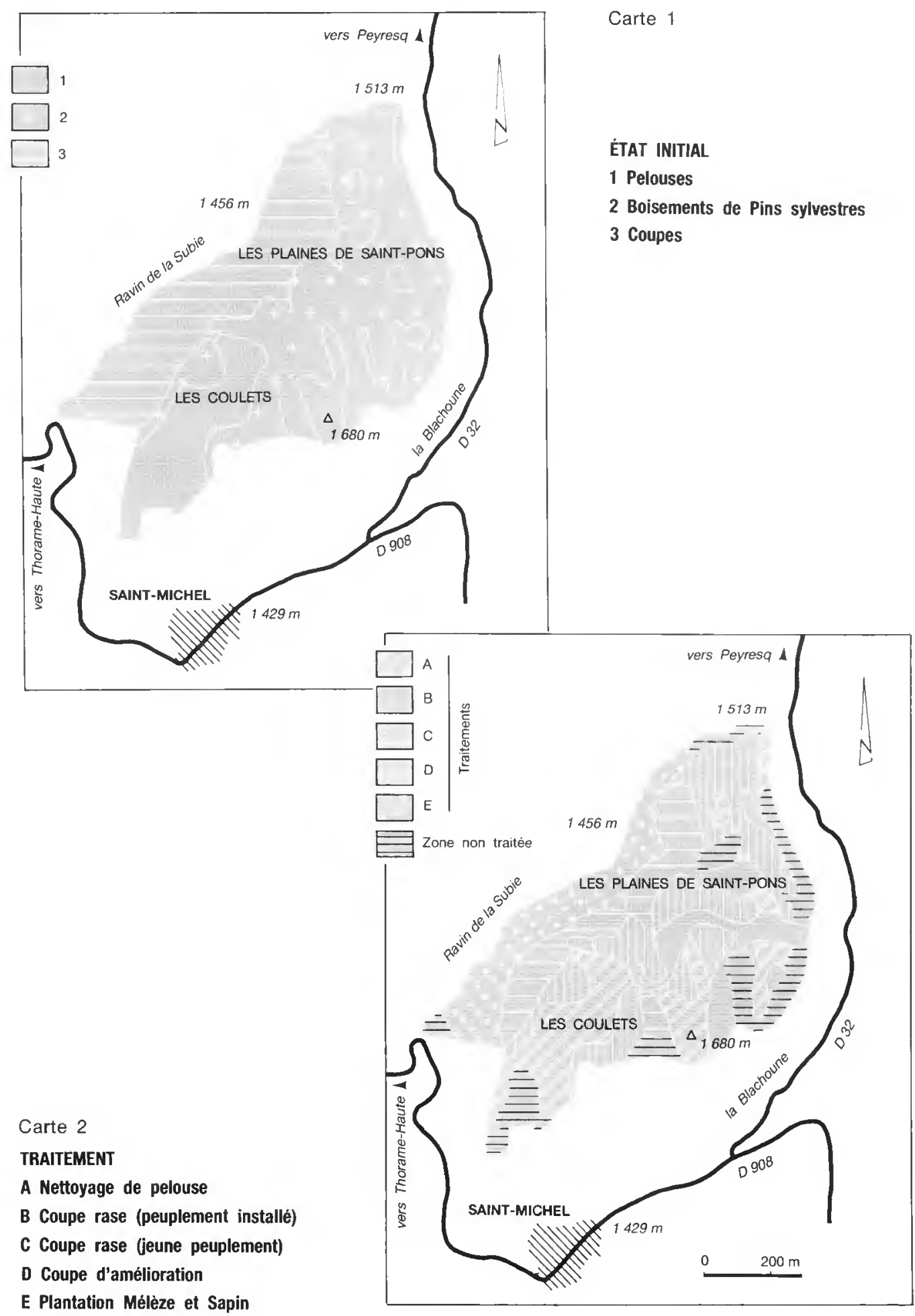



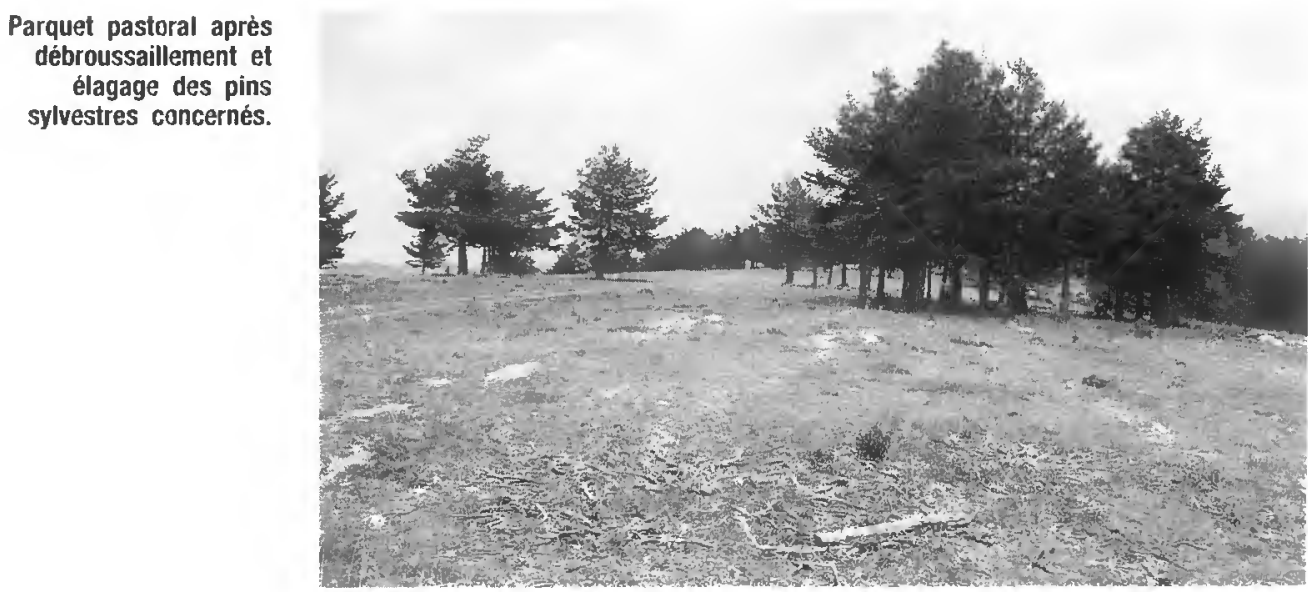

Parquet forestier après

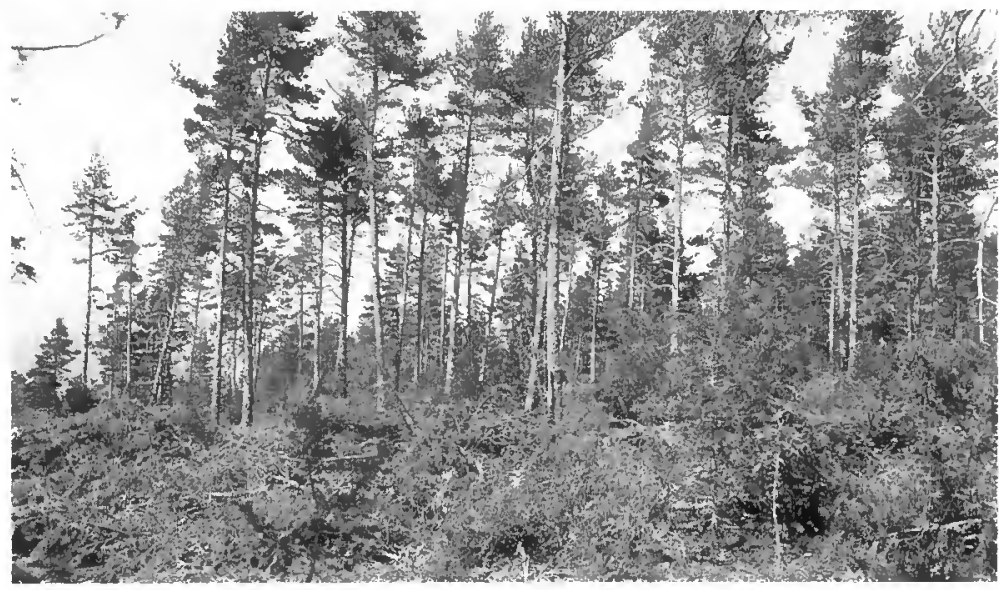

Photos G. DECAIX exploitation et avant broyage des rémanents.

classique par parquets mais où certains parquets ne sont pas boisés. Âge d'exploitabilité : 100 ans environ; dépressage dans les jeunes peuplements (10-15 ans) laissant environ 1100 tiges/ha ; trois coupes d'éclaircie à 40,60 et 80 ans ramenant la densité autour de 400-500 tiges/ha ; coupe d'ensemencement forte laissant 200 à 250 semenciers/ha suivie au maximum d'une secondaire mais le plus souvent d'une coupe définitive.

Le volet pastoral, à travers une convention pluriannuelle avec l'éleveur, impose le gardiennage en parcs clôturés selon un plan prédéfini et un calendrier d'utilisation basé sur la ressource pastorale et la phénologie des espèces, ainsi que l'entretien des équipements et le débroussaillage des recrus éventuels. La contrepartie à ces contraintes pour l'éleveur consiste en un gain de temps appréciable. Une intervention quotidienne pour l'abreuvement du troupeau, voire bi-hebdomadaire (quand l'eau peut être distribuée dans chaque parc), suffit à surveiller la santé du cheptel, l'état des clôtures et l'avancement de la consommation d'herbe dans le parc.

L'utilisation touristique est garantie par l'entretien des pistes, la mise en place de passages aménagés au niveau des clôtures, la réglementation des exploitations forestières (périodes d'interdiction, respect des clôtures, remise en état des sentiers...). De plus, l'aménagement sylvopastoral est utilisé comme outil pédagogique (à travers un sentier d'interprétation) présentant les problèmes de gestion de l'espace rural. 


\section{G. DECAIX}

\section{LES ÉVOLUTIONS RÉCENTES}

Depuis la mise en œuvre de la phase de gestion à La Colle Saint-Michel en 1988, plusieurs autres aménagements sylvopastoraux ont vu progressivement le jour à Thorame-Basse, Vergons, Angles, Lambruisse. Ils ont permis d'affiner et d'adapter, à d'autres conditions, la conception initiale de ce type d'exploitation.

\section{Au niveau des traitements}

À côté des parquets strictement pastoraux ou forestiers, se sont insérés des parquets de pâturages arborés. Ils ont été installés là où la qualité initiale du boisement ne peut laisser espérer une véritable production forestière, mais où le maintien du Pin sylvestre à la densité de 80 à 100 tiges par hectare assure un couvert qui retarde le dessèchement de la strate herbacée, un meilleur confort pour les animaux et une activité mieux soutenue. Ainsi, dans des conditions écologiques difficiles, peut-on étaler la période d'utilisation par le troupeau pendant une partie de l'été et éventuellement éviter la location d'un pâturage d'estive.

Dans ce type de traitement, après l'abattage des arbres et leur extraction, on procéde à l'élagage des tiges restantes puis au broyage des broussailles et de la totalité des rémanents pour un coût de 6500 à $7000 \mathrm{~F} / \mathrm{ha}$.

\section{Au niveau des techniques}

L'incinération des rémanents, qui nécessite de nombreuses manipulations et ne peut être réalisée en période de risque d'incendie, a été abandonnée au profit du broyage.

Les broyeurs à marteaux portés sur un tracteur à roues de 240 chevaux ou sur le bras d'une pelle donnent des résultats supérieurs à l'incinération pour un coût variant de 4000 à $6000 \mathrm{~F} / \mathrm{h}$ a selon la topographie et l'importance des rémanents. Portés par une pelle, ils permettent également de broyer certaines tiges sur pied.

\section{Au niveau de l'impact paysager et de l'utilisation touristique}

Si à La Colle Saint-Michel aucune étude paysagère n'a été faite, l'imbrication des parquets pastoraux et forestiers offrent toutefois un site très apprécié des skieurs et des randonneurs.

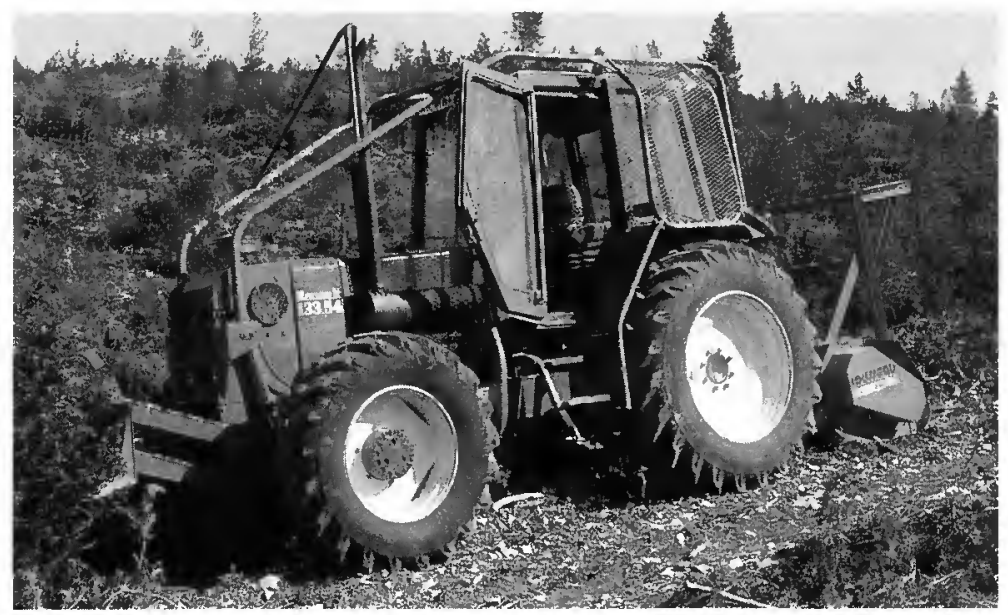

1 Tracteur et broyeur.

Clôture électrique 4 fils : remarquer, à droite, la zone påturée avec herbe rase, et à gauche, la zone non pâturée avec herbe haute.

Photos G. DECAIX 


\section{Le sylvopastoralisme}

Les nouveaux projets, par contre, intègrent dès le départ des préoccupations paysagères. À Layon, sur la commune de Thorame-Basse, la répartition comme la forme des coupes ont fait l'objet d'une modélisation préalable permettant de tester plusieurs scénarios. Confrontés aux contraintes topographiques, à la nature des peuplements et aux potentialités pastorales, en est dégagé un compromis.

À Layon toujours, le site rénové servant aussi aux aires d'envol des parapentes et deltaplanes, des aménagements mineurs ont permis de concilier les différentes activités : installation des clôtures en fonction des aires d'envol, mise en place de "passages canadiens" adaptés aux ovins supprimant les problèmes de porte, etc...

\section{EN GUISE DE CONCLUSION : QUELQUES ÉLÉMENTS INDISPENSABLES À LA RÉUSSITE}

Après dix ans d'expérience, la palette des traitements possibles ainsi que les techniques de rénovation sont assez bien cernées. Les surfaces aménagées couvrant plus de 700 ha et étant assez bien représentatives d'une large gamme de situations, seules des adaptations mineures peuvent être envisagées. Pourtant, étendre ce type de mise en valeur aux milliers d'hectares d'anciens parcours ovins, qui couvrent les Préalpes du Sud, nécessite de prendre certaines précautions pour éviter un échec.

\section{Définir clairement les objectifs et les actions qui en découlent}

Une analyse détaillée de l'état initial permettant de cerner les potentialités forestières et pastorales mais aussi les contraintes de protection, de paysage et d'accueil du public, est indispensable.

En la confrontant aux besoins exprimés par les propriétaires et les utilisateurs potentiels des sites rénovés, on doit faire ressortir clairement les objectifs et donc les actions de remise en état à entreprendre.

\section{Garantir le suivi de gestion}

L'investissement initial est toujours important pour un revenu direct (production forestière et location du pâturage) réduit. II convient donc que toutes les précautions soient prises pour assurer la gestion et l'entretien du site.

Le gardiennage en parcs clôturés doit être obligatoire. C'est en effet le meilleur moyen de maintenir
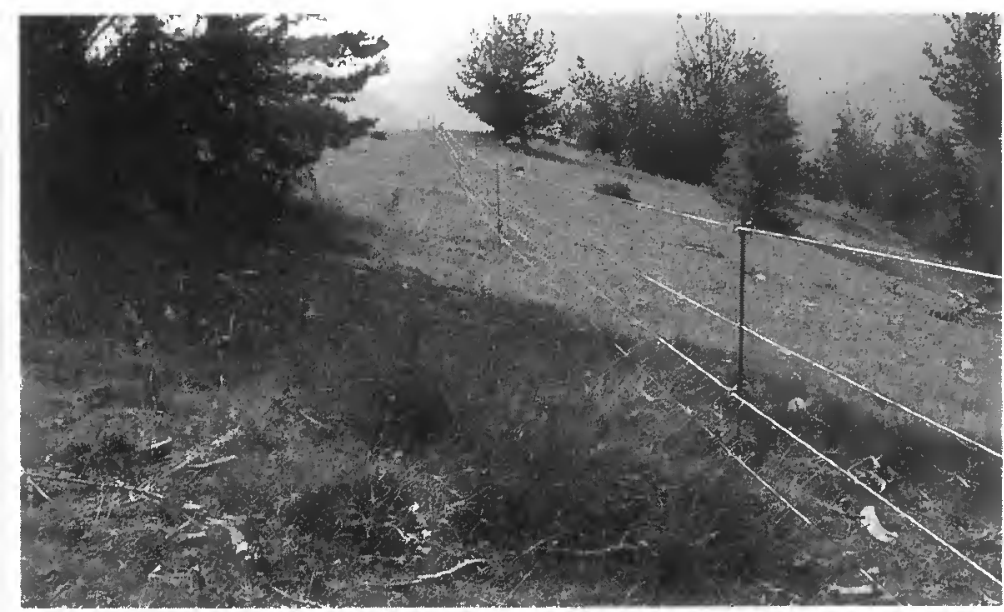
des charges pastorales permettant l'utilisation optimale de la strate herbacée et limitant les rejets de la strate arbustive et donc les entretiens ultérieurs.

Un contrat de gestion fixant les droits et les devoirs du propriétaire et de l'éleveur est aussi indispensable. II doit préciser, en particulier, l'importance du troupeau, la période d'utilisation, le calendrier des parcours et la charge pastorale sur chaque parc, les conditions d'entretien des équipements et de réalisation des débroussaillements complémentaires. 


\section{G. DECAIX}

\section{La concertation entre les différents partenaires}

De ce qui précède, il ressort à l'évidence que la concertation entre les différents partenaires est une nécessité indispensable dès la phase initiale du projet.

Le propriétaire et le gestionnaire doivent définir, avec les techniciens pastoraux, le profil des éleveurs en fonction du type de remise en valeur retenue. De la discussion avec les éleveurs se précisent les possibilités de gestion. Ainsi le projet s'affine progressivement; le délai moyen pour la définition et la mise en place des cinq projets sylvopastoraux entrepris a été de six mois et deux années respectivement.

L'animation semble toujours devoir être menée par les techniciens pastoraux qui ont, de loin, la meilleure approche du milieu professionnel. Inscrite dans leur activité quotidienne, elle reste difficile à chiffrer. 